\title{
Antibiotic susceptibilities of Yersinia enterocolitica and $Y$. intermedia isolates from aquatic environments
}

\author{
E. TZELEPI, M. ARVANITIDOU*, A. MAVROIDI and A. TSAKRIS ${ }^{\dagger}$ \\ Department of Bacteriology, Hellenic Institute Pasteur, Athens and Departments of *Hygiene and \\ ${ }^{\dagger}$ Microbiology, Medical School, Aristotelian University of Thessaloniki, Greece
}

\begin{abstract}
Of 37 Yersinia isolates from various aquatic environments, seven were $Y$. enterocolitica and $30 \mathrm{Y}$. intermedia. These isolates were biotyped, serotyped and tested for their susceptibility to 20 antibiotics. All $Y$. enterocolitica isolates were of biovar 1; those of $Y$. intermedia were distributed amongst four biovars (1, 2, 4 and 6). On the basis of combined biotyping and serotyping results, $Y$. enterocolitica isolates were distributed in five and $Y$. intermedia in 17 groups. With the exception of one $Y$. enterocolitica isolate which was resistant to tetracycline and streptomycin, the isolates were sensitive to the non- $\boldsymbol{\beta}$-lactam antibiotics. In contrast, various patterns of $\boldsymbol{\beta}$-lactam insensitivity were detected, including ampicillin and ticarcillin (35 isolates), cephalothin (33 isolates), carbenicillin (32 isolates), amoxycillin/clavulanate ( 23 isolates) and cefoxitin ( 22 isolates). No correlation between biotype or serotype and the susceptibility pattern of the isolates was apparent. Both inducible cephalosporinase activity against third-generation cephalosporins and inhibition of resistance to penicillins were detected in all $Y$. enterocolitica and $Y$. intermedia isolates by double-disk tests.
\end{abstract}

\section{Introduction}

Yersiniosis is related to various clinical syndromes, mainly caused by Yersinia enterocolitica, although all Yersinia species have been isolated from clinical specimens [1]. The micro-organism is usually transmitted by ingestion of contaminated food. Water supplies may also contribute to the dissemination of the disease. Yersinia strains are highly adaptable to aquatic environments, having minimal nutrient requirements and being capable of surviving in cold water for long periods [2]. Thus, surface water contaminated by sewage discharges may be a significant vehicle for transmission of human yersiniosis.

Antimicrobial susceptibilities have been documented sporadically for Yersinia strains of human, animal or food origin [3-6]. In these reports, yersiniae were usually found to be susceptible to most antibiotics, although a considerable number of strains showed decreased susceptibility to some older $\beta$-lactams. Published studies on $\beta$-lactamases focus on $Y$. enterocolitica, where two distinct chromosomal enzymes

Received 16 Feb. 1998; revised version received 6 May 1998; accepted 22 June 1998.

Corresponding author: Dr A. Tsakris. (named A and B) have been characterised [7-9]. However, there is a paucity of data concerning antibiotic susceptibility among strains of Yersinia isolated from aquatic environments. Furthermore, reports on susceptibility profiles of the previously named ' $Y$. enterocolitica-like' species, and in particular of $Y$. intermedia, are rare.

This study reports the occurrence of $Y$. enterocolitica and $Y$. intermedia in aquatic environments of Greece. Data on the susceptibility of the isolates to a wide range of antibiotics are also presented.

\section{Materials and methods}

\section{Samples and isolation procedures}

Of 37 Yersinia isolates from samples taken from various aquatic environments in Northern Greece, nine came from three distinct bathing areas (Thessaloniki and Platamon in the Thermaikos Gulf, and Marmaras in the Toroneos Gulf, Chalkidiki), seven from river water samples (from the rivers Axios, Aliakmon and Strimon), 18 from drinking water samples in various areas and three were from mussels harvested from sea-water.

Yersinia spp. were isolated from water samples as 
described previously [10]. Isolation of yersiniae from mussels involved homogenisation of mussels (30$40 \mathrm{~g})$ in sterile distilled water $(120-160 \mathrm{ml})$ in a Vir Tis blender at c. $50000 \mathrm{rpm}$ for $1 \mathrm{~min}$. The blended mussel suspension $(50 \mathrm{ml})$ was mixed with an equal volume of double-strength Yersinia enrichment medium and incubated at $4^{\circ} \mathrm{C}$ for 21 days. One loopful of this cold mixture was inoculated on to CefsulodinIrgasan-Novobiocin (CIN) Agar (Oxoid) and incubated at $32^{\circ} \mathrm{C}$ overnight. Examination of the suspected colonies and identification of the Yersinia isolates was performed as for the water samples. Biotyping and serotyping based on somatic antigens were performed by $\mathrm{H}$. H. Mollaret (National Centre of Yersiniae, Institute Pasteur, Paris, France).

\section{Antibiotic sensitivity testing}

Yersinia isolates were tested for their susceptibility to 20 antimicrobial agents: ampicillin, ticarcillin, amoxycillin/clavulanate, piperacillin, carbenicillin, cephalothin, cefoxitin, cefuroxime, ceftazidime, cefotaxime, aztreonam, imipenem, streptomycin, spectinomycin, gentamicin, tobramycin, amikacin, tetracycline, chloramphenicol and co-trimoxazole. Susceptibility tests were performed at $28^{\circ} \mathrm{C}$ by a standard disk diffusion method, with Mueller-Hinton agar and antibiotic disks from Diagnostics Pasteur (Marnes La Coquette, France). Results were recorded by measuring the inhibition zones and scored as sensitive, intermediate susceptibility and resistant according to the NCCLS recommendations [11]. Escherichia coli ATCC 25922 was used as a reference strain.

\section{Screening for induction and inhibition of $\beta$ - lactamase expression}

To detect inducible cephalosporinase activity, a double- disk antagonism test was performed as described by Sanders et al. [12]. A disk of imipenem $(30 \mu \mathrm{g})$ was placed near to disks containing ceftazidime $(30 \mu \mathrm{g})$ and cefotaxime $(30 \mu \mathrm{g})$. Cephalosporinase activity was reflected by a decrease in inhibition radius around the disks of third-generation cephalosporins placed next to the disk of imipenem, which is a stronger inducer. Inhibition of penicillinase activity was detected by a double-disk synergy test, as described by Jarlier et al. [13]. A disk of amoxycillin + clavulanate $(20 \mu \mathrm{g}+$ $10 \mu \mathrm{g})$ was placed near disks of carbenicillin $(100 \mu \mathrm{g})$, ampicillin $(10 \mu \mathrm{g})$ and ticarcillin $(75 \mu \mathrm{g})$ [13]. Synergy between the disk containing the $\beta$ lactamase inhibitor and those containing penicillins revealed penicillinase activity.

\section{Results}

Of 37 Yersinia isolates from the aquatic samples examined, 30 were identified as $Y$. intermedia and seven as $Y$. enterocolitica. Of the nine isolates from sea-water, seven were identified as $Y$. intermedia and two as $Y$. enterocolitica. All seven isolates from rivers were $Y$. intermedia. Of the isolates from drinking water, 15 were $Y$. intermedia and three $Y$. enterocolitica, while two $Y$. enterocolitica and one $Y$. intermedia were isolated from mussels. All $Y$. enterocolitica isolates belonged to biovar 1 , whereas $Y$. intermedia isolates belonged to four different biovars with biovar 2 being the most common (12 isolates). When biotyping and serotyping were considered in combination, $Y$. enterocolitica isolates were distributed in five groups and $Y$. intermedia isolates in 17 groups (Table 1).

All the isolates were susceptible to the non- $\beta$-lactam antibiotics tested, with the exception of one $Y$.

Table 1. Characteristics of seven $Y$. enterocolitica and $30 \mathrm{Y}$. intermedia isolates

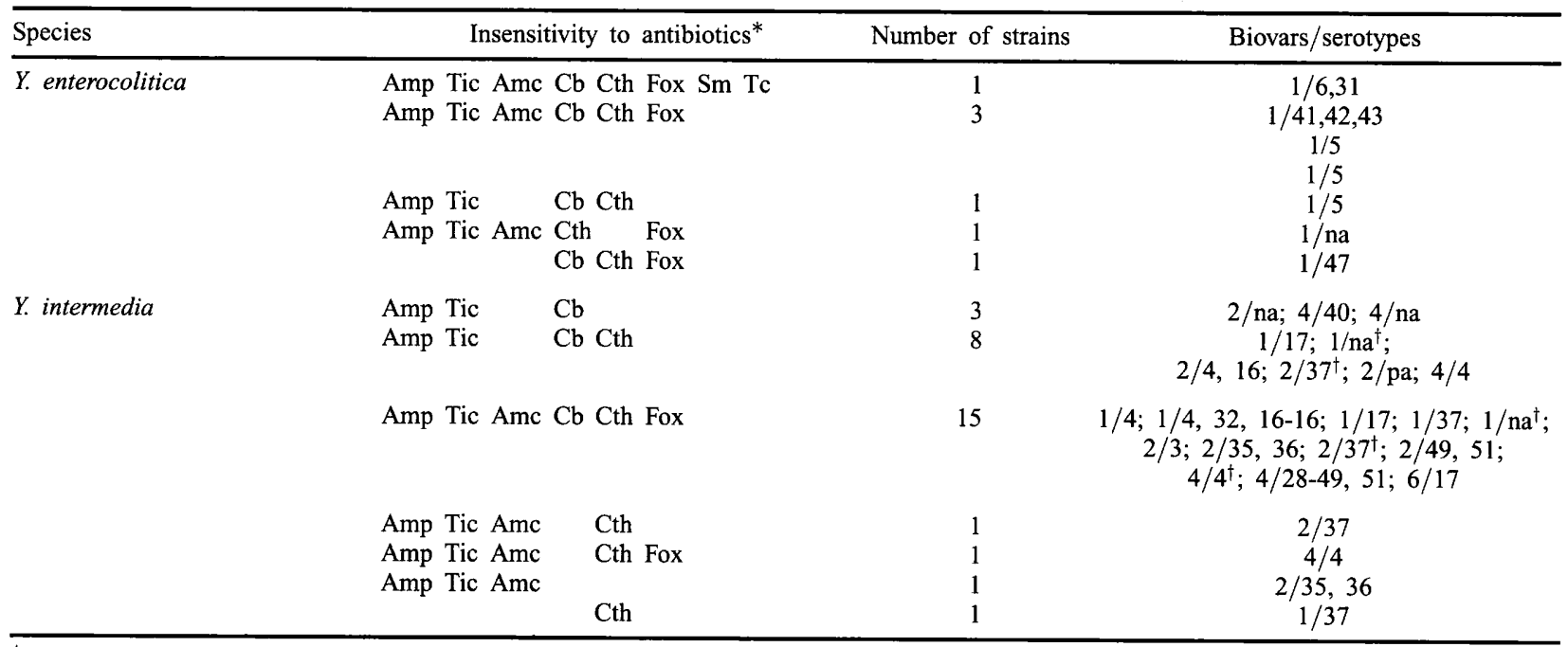

* The term insensitivity was used to include resistance and intermediate susceptibility. Antibiotics: Amp, ampicillin; Tic, ticarcillin; Amc, amoxycillin+clavulanate; $\mathrm{Cb}$, carbenicillin, Cth, cephalothin; Fox, cefoxitin; Sm, streptomycin; Tc, tetracycline; na, non-agglutinable; pa, polyagglutinable.

${ }^{\dagger}$ Type exhibited by two strains in this group. 
Table 2. Susceptibility* of seven $Y$. enterocolitica and $30 \mathrm{Y}$. intermedia isolates to $12 \beta$-lactam antibiotics

\begin{tabular}{|c|c|c|c|c|c|c|}
\hline \multirow[b]{2}{*}{ Antimicrobial agent } & \multicolumn{3}{|c|}{ Y. enterocolitica } & \multicolumn{3}{|c|}{$Y$. intermedia } \\
\hline & Sensitive & Intermediate & Resistant & Sensitive & Intermediate & Resistant \\
\hline Ampicillin & 1 & 0 & 6 & 1 & 18 & 11 \\
\hline Amoxycillin/clavulanate & 2 & 0 & 5 & 12 & 16 & 2 \\
\hline Ticarcillin & 1 & 0 & 6 & 1 & 18 & 11 \\
\hline Piperacillin & 7 & 0 & 0 & 30 & 0 & 0 \\
\hline Carbenicillin & 1 & 2 & 4 & 4 & 1 & 25 \\
\hline Cephalothin & 0 & 2 & 5 & 4 & 6 & 20 \\
\hline Cefoxitin & 1 & 5 & 1 & 14 & 14 & 2 \\
\hline Cefuroxime & 7 & 0 & 0 & 30 & 0 & 0 \\
\hline Ceftazidime & 7 & 0 & 0 & 30 & 0 & 0 \\
\hline Cefotaxime & 7 & 0 & 0 & 30 & 0 & 0 \\
\hline Aztreonam & 7 & 0 & 0 & 30 & 0 & 0 \\
\hline Imipenem & 7 & 0 & 0 & 30 & 0 & 0 \\
\hline
\end{tabular}

* Susceptibility status was defined according to NCCLS criteria [11]

enterocolitica isolate which was resistant to tetracycline and streptomycin. The results of susceptibility testing for $\beta$-lactam agents are presented in Tables 1 and 2. Marked antibacterial activity was shown with piperacillin, as well as with third generation cephalosporins (ceftazidime and cefotaxime), aztreonam and imipenem. Of the second-generation cephalosporins tested, cefuroxime was active against all the isolates, whereas cefoxitin exhibited mostly moderate activity. The majority of the isolates were either resistant or intermediately susceptible to the older $\beta$-lactam antibiotics (ampicillin, ticarcillin, carbenicillin and cephalothin); most of them were also insensitive to the amoxycillin/clavulanate combination. No correlation between biotype or serotype and the susceptibility pattern of the isolates was apparent. However, in double-disk tests, inhibition of penicillinase activity between amoxycillin/clavulanate and ampicillin or carbencillin or ticarcillin disks was visible in all cases, even in one isolate that was sensitive to all penicillins. Also, induction of resistance to thirdgeneration cephalosporins by imipenem was always present.

\section{Discussion}

Yersinia spp. have emerged as pathogens associated with various infections in man, but little is known about the occurrence and antimicrobial susceptibility of strains encountered in aquatic environments. It appears that $Y$. intermedia is the predominant species isolated from aquatic samples in Northern Greece; Y. enterocolitica was found less frequently. This is in contrast to previous findings from Italy, reporting $Y$. enterocolitica as the prevalent species isolated from river water [14]. Furthermore, unlike the latter study, other neighbouring species, such as $Y$. frederiksenii and $Y$. kristensenii, were not identified in the study region. It is of interest that none of the isolates was in a biogroup or serogroup that has traditionally been associated with human disease. However, Y. enterocolitica biotype 1, serotype $0: 5$, one of the prevalent subgroups in the present study, has previously been associated with gastrointestinal infection [15], while there is an increasing body of data suggesting that strains outside standard pathogenic groups or species can cause illness in $\operatorname{man}[1,16]$.

Susceptibility results showed a very low prevalence of resistance to non- $\beta$-lactam antibiotics among Yersinia isolates. This finding is in agreement with those of other authors $[4,6,17]$. It has been proposed that low resistance rates reflect the poor ability of Yersinia spp. to act as recipients in naturally occurring bacterial conjugation [4]. With respect to $\beta$-lactam antibiotics, most of the isolates were insensitive to the older $\beta$ lactam agents, including carbenicillin, and considerable numbers of them were also insensitive to the amoxycillin/clavulanate combination and to cefoxitin. It has been stated that strains of less human-related serotypes may be more frequently susceptible to ampicillin and cephalothin [3,5]. Nevertheless, the results of the present study did not differ essentially from those reported by other authors, who examined yersiniae isolated from foods as well as from human and animal infections. In those studies, almost all $Y$. enterocolitica biovar 1 and $Y$. intermedia strains were resistant to ampicillin, cephalothin and cefoxitin $[4,6]$.

In $Y$. enterocolitica, resistance to penicillins and older cephalosporins has been attributed to the production of $\beta$-lactamases [5]. Cornelis and Abraham [7] studied the $\beta$-lactamases produced by a number of $Y$. enterocolitica strains of human and animal origin extensively and characterised two chromosomally mediated enzymes - a constitutive penicillinase named enzyme $A$ and an inducible cephalosporinase they named enzyme B. Pham et al. [8] also studied $\beta$ lactamases of $Y$. enterocolitica isolates from Australia and found that biotype 1 strains produced both $\beta$ lactamases, in contrast with strains of biotypes 3 and 4 which were found to possess only one type of $\beta$ lactamase. However, there are no reports describing $\beta$ lactamases in $Y$. intermedia. Results from the doubledisk tests in the present study indicate that strains in 
this species also produce $\beta$-lactamases of both types. Induction of resistance to third-generation cephalosporins by imipenem indicated the presence of inducible cephalosporinases in all the $Y$. intermedia and $Y$. enterocolitica isolates in the present study. Similarly, inhibition of growth between clavulanatecontaining disks and disks of penicillins indicated that all strains might also produce penicillinases.

In a previous report, differences in susceptibility to $\beta$ lactam agents within the same $Y$. enterocolitica biotype were found, when isolates came from distinct countries [9]. Isolates belonging to the same biovar and serotype but differing in $\beta$-lactam susceptibility phenotypes were also found in the present study in both Yersinia species examined. The majority of the isolates exhibited broad patterns of insensitivity, including resistance to both penicillins and cephalosporins. This is in agreement with previous studies, in which the simultaneous presence of a penicillinase and a cephalosporinase has been related to cross-resistance to penicillins, amoxycillin/clavulanate, cephalothin and cefoxitin [7,8]. Resistance to cefoxitin has previously been found only in clinical $Y$. enterocolitica strains of biovar 1. [17]. Interestingly, all but one of the $Y$. enterocolitica and more than half of the $Y$. intermedia isolates in this study were found to be insensitive to cefoxitin. These observations suggest that the aquatic environment may contribute to the spread of yersiniae expressing resistance to various $\beta$ lactam antibiotics.

\section{References}

1. Sulakvelidze A, Dalakishvili K, Barry E et al. Analysis of clinical and environmental Yersinia isolates in the republic of Georgia. $J$ Clin Microbiol 1996; 34: 2325-2327.

2. Schiemann DA. Yersinia enterocolitica in drinking water. In: McFeters GA (ed) Drinking water microbiology. Brock/Springer Series in Contemporary Bioscience. New York, Springer Verlag. 1990: 322-339.
3. Juhlin I, Winblad S. Susceptibility to mecillinam and other antibiotics of 28 O-serotypes of Yersinia enterocolitica. $J$ Antimicrob Chemother 1981; 8: 291-297.

4. Ahmedy A, Vidon DJ-M, Delmas CL, Lett M-C. Antimicrobial susceptibilities of food-isolated strains of Yersinia enterocolitca, $Y$. intermedia, $Y$. frederiksenii, and Y. kristensenii. Antimicrob Agents Chemother 1985; 28: 351-353.

5. Hornstein MJ, Jupeau AM, Scavizzi MR, Philippon AM, Grimont PAD. In-vitro susceptibilities of 126 clinical isolates of Yersinia enterocolitica to $21 \beta$-lactam antibiotics. Antimicrob Agents Chemother 1985; 27: 806-811.

6. Preston MA, Brown S, Borczyk AA, Riley G, Krishnan C. Antimicrobial susceptibility of pathogenic Yersinia enterocolitica isolated in Canada from 1972 to 1990. Antimicrob Agents Chemother 1994: 38: 2121-2124.

7. Cornelis G, Abraham EP. $\beta$-Lactamases from Yersinia enterocolitica. J Gen Microbiol 1975; 87: 273-284.

8. Pham JN, Bell SM, Lanzarone JYM. A study of the $\beta$ lactamases of 100 clinical isolates of Yersinia enterocolitica. $J$ Antimicrob Chemother 1991; 28: 19-24.

9. Pham JN, Bell SM, Hardy MJ, Martin L, Guiyoule A, Carniel E. Susceptibility to $\beta$-lactam agents of Yersinia enterocolitica biotype 4 , serotype $\mathrm{O}: 3$ isolated in various parts of the world. $J$ Med Microbiol 1995; 43: 9-13.

10. Arvanitidou M, Stathopoulos GA, Constantinidis TC, Katsouyannopoulos V. The occurrence of Salmonella, Campylobacter and Yersinia spp. in river and lake waters. Microbiol Res 1995; 150: 153-158.

11. National Committee for Clinical Laboratory Standards. Performance Standards for Antimicrobial Disc Susceptibility Tests; M2A2. Villanova, PA, NCCLS. 1992.

12. Sanders CC, Sanders WE, Goering RV. In vitro antagonism of beta-lactam antibiotics by cefoxitin. Antimicrob Agents Chemother 1982; 21: 968-975.

13. Jarlier V, Nicolas MH, Fournier G, Philippon A. Extended broad-spectrum $\beta$-lactamases conferring transferable resistance to newer $\beta$-lactam agents in Enterobacteriaceae: hospital prevalence and susceptibility patterns. Rev Infect Dis 1988; 10: $867-878$.

14. Massa S, Cesaroni D, Poda G, Trovatelli LD. Isolation of Yersinia enterocolitica and related species from river water. Zentralbl Microbiol 1988; 143: 575-581.

15. Ratnam S, Mercer E, Picco B, Parsons S, Butler R. A nosocomial outbreak of diarrheal disease due to Yersinia enterocolitica serotype 0:5, biotype 1. Infect Dis 1982; 145: 242-247.

16. Bissett ML, Powers C, Abbot SL, Janda JM. Epidemiologic investigations of Yersinia enterocolitica and related species: sources, frequency, and serogroup distribution. J Clin Microbiol 1990; 28: 910-912.

17. Pham JN, Bell SM, Lanzarone JYM. Biotype and antibiotic sensitivity of 100 clinical isolates of Yersinia enterocolitica. $J$ Antimicrob Chemother 1991; 28: 13-18. 